\title{
UROLOGICAL CANCER
}

\section{Heart facts rehabilitate ADT}

Androgen-deprivation therapy (ADT) is widely used for the treatment of patients with prostate cancer, but has been suggested to increase a patient's risk of dying from cardiovascular complications. However, in a meta-analysis of eight prospective, randomized trials with over 4,000 patients, a team of researchers based at Harvard found no evidence that ADT increases the risk of cardiovascular death of patients with aggressive prostate cancer-a finding lead investigator Paul L. Nguyen calls "reassuring”.

Results from various studies (mainly retrospective) indicated a link between ADT and cardiovascular adverse effects, which prompted a joint statement from several American medical societies and even called the FDA into action. "Because retrospective studies can be open to confounding and selection bias" explains Nguyen, "we wanted to see if the randomized data also found an association between the two."

His team identified eight prospective, randomized multicenter phase III trials of patients with nonmetastatic and non-hormone-refractory disease that had an intervention group with ADT and appropriate control groups. The median follow-up ranged from 7.6 years to 13.2 years. Cardiovascular death was a separate end point in all the studies.

The combined data indicated that patients who received ADT were not more likely to die from cardiovascular causes than those in the control group (RR [relative risk] 0.93). Similarly, the investigators found no excess cardiovascular mortality owing to ADT in several subgroups, including patients who received short-course ADT (6 months), long-course ADT ( $\geq 3$ years), concomitant radiotherapy, or who were older than 70 years at enrollment. Importantly, ADT decreased the risk of death owing to prostate cancer (RR 0.69) and all-cause mortality (RR 0.86).

One of the limitations of the metaanalysis is that the team could not tell whether cardiovascular events occured earlier if patients received ADT, as only the cumulative incidence was reported. Furthermore, as none of the trials was stratified for existing cardiovascular disease, the study cannot exclude the

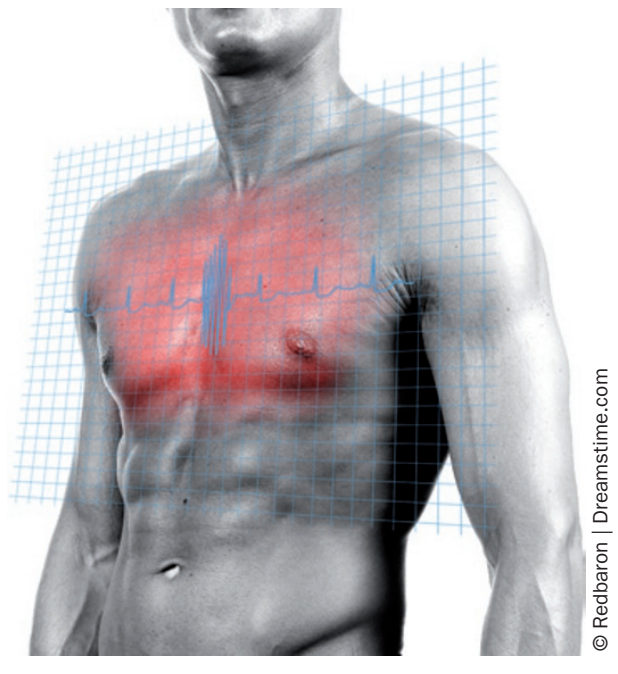

possibility that patients with this type of prostate cancer and pre-existing cardiovascular problems (approximately $5-10 \%$, according to Nguyen) could still be harmed by ADT. The investigators therefore recommend that patients be stratified by cardiovascular comorbidities in future randomized trials of ADT.

Christoph A. Schmitt

Original article Nguyen, P. L. et al. Association of androgen deprivation therapy with cardiovascular death in patients with prostate cancer. JAMA 306, 2359-2366 (2011) 